\title{
EFFECTS OF A COMBINATION THERAPY WITH ATORVASTATIN AND METFORMIN ON THE GLYCEMIC CONTROL AND ADIPOSITY INDICES IN NEWLY DIAGNOSED OVERWEIGHT PATIENTS WITH TYPE 2 DIABETES MELLITUS: A PILOT STUDY
}

\author{
SAMER SHUKUR MOHAMMED, WAEL WALEED MUSTAFA, SAAD ABDULRAHMAN HUSSAIN* \\ Department of Pharmacology and Toxicology, Faculty of Pharmacy, Al-Rafidain University College, Baghdad, Iraq. \\ Email: saad.hussain@coalrafidain.edu.iq
}

Received: 05 July 2018, Revised and Accepted: 30 July 2018

\section{ABSTRACT}

Objective: In many type 2 diabetes mellitus (T2DM) patients, metformin is prescribed concomitantly with hypolipidemic agents, particularly statins. Meanwhile, variability in response to metformin is one of the most important problems in the efficacy of this combination. The present study aims to evaluate the effect of adding atorvastatin with metformin on the glycemic control, adiposity indices, and lipid profile of overweight patients newly diagnosed with type T2DM.

Methods: A total of 50 overweight patients with T2DM were allocated into two groups, the first one received $850 \mathrm{mg} / \mathrm{day}$ of sustained release metformin and the second group received $10 \mathrm{mg} /$ day atorvastatin in addition to the metformin. The patients were followed for 90 days through evaluating fasting serum glucose (FSG), glycated hemoglobin (HbA1c), body mass index (BMI), visceral adiposity index (VAI), and the lipid profile at baseline and after 90 days. In addition, the safety of the protocol was monitored through the evaluation of the renal and liver functions.

Results: HbA1c, FSG, BMI, and VAI values were significantly decreased in both treatment groups compared with baseline. Meanwhile, the combination improves all the lipid profile components with respect to the baseline. No significant differences reported between the two groups regarding all the measured parameters. The addition of atorvastatin produced a slight but significant negative impact on the renal and liver functions.

Conclusion: Addition of $10 \mathrm{mg} /$ day atorvastatin with metformin in the treatment of newly diagnosed T2DM overweight patients did not produce significant improvement in glycemic control, adiposity index, and lipid profile compared with the use of metformin alone.

Keywords: Metformin, Atorvastatin, Type 2 diabetes mellitus, Adiposity index, Glycemic control.

(c) 2018 The Authors. Published by Innovare Academic Sciences Pvt Ltd. This is an open access article under the CC BY license (http://creativecommons. org/licenses/by/4. 0/) DOI: http://dx.doi.org/10.22159/ajpcr.2018.v11i12.28309

\section{INTRODUCTION}

Many studies have concluded that the prescribing practice during the treatment of type 2 diabetes mellitus (T2DM) focused on the use of a combination therapy to target most of the associated risk factors $[1,2]$. The therapeutic benefits of statins as hypolipidaemic agents are recognized not only in patients with dyslipidemia $[3,4]$ but also in patients early diagnosed or already having glucose metabolism disorders [5,6]. This may lead to the suggestion that patients early diagnosed with T2DM may benefit the use of statins, like atorvastatin, as "add-on" treatment with metformin in the treatment of patients suffering from impaired glucose metabolism compared with the use of metformin alone. The pathophysiological processes of T2DM may negatively impact the plasma lipids and lipoproteins, and the patients with T2DM often complain of dyslipidemia that enhances the risk of cardiovascular diseases (CVDs) [7,8]. Many reports indicated that around $80 \%$ of T2DM patients die due to its cardiovascular complications (CVD) [9], and the adjunct use of statins may reduce the CVD risks in those patients [10] and recommend early administration of these lipid-lowering agents in diabetic patients to prevent CVD mortality [11]. In newly diagnosed T2DM cases, the American Diabetes Association recommends changing lifestyle and starting metformin therapy [12]. Metformin has pleiotropic effects and can improve lipid metabolism and vascular function; although the inconsistent response to metformin was problematic in the efficacy of this drug [13] and other drugs such as statins may be added to the treatment protocol. However, accumulating evidence indicates that concomitant use of statins may negatively impact glycemic control and worsen the symptoms of T2DM [14]. We, therefore, performed a pilot clinical study to evaluate the effect of adding a low dose of atorvastatin with the prescribed dose of metformin on the treatment outcome of overweight newly diagnosed patients with T2DM.

\section{METHODS}

\section{Patients and study design}

A total of 50 newly diagnosed patients with T2DM with age range of $47.8 \pm 7.4$ years and prescribed a sustained release $850 \mathrm{mg} /$ day of metformin, after inappropriate management with dietary restriction, were enrolled in this open-label multicenter pilot study. All patients were newly diagnosed according to the WHO criteria [15] and underwent a physical examination and information about their medical history, demographic parameters, and medication history were obtained by questionnaire. The exclusion criteria include patients with T1DM, previous history of renal failure, autoimmune and liver diseases, serious chronic disorders, and pregnancy. The patients were randomly allocated into two groups (25 patients each) and followed for 90 days in this pilot study. During this period, the first group received $850 \mathrm{mg}$ /day metformin (Merck Santé SAS, Lyon, France), while the second group consumed $10 \mathrm{mg} /$ day of atorvastatin (Pfizer Ltd, Kent, UK) with the same metformin dose of the first group. Of 50 participants, 17 were female and 33 were male. The patients received a constant dose regimen of metformin during the 90-day study period. In addition, few of them were already prescribed other drugs such as aspirin, calcium channel blockers, angiotensin-converting enzyme inhibitors, and angiotensin receptor blockers (Table 1). The study protocol was approved by the local Ethics Committee at Al-Rafidain University College in accordance with the principles of the Helsinki Declaration. All the enrolled patients provided written informed consent to participate in this pilot study. 
Table 1: Baseline characteristics of the T2DM patients

\begin{tabular}{llll}
\hline Parameters & Met $\mathbf{n = 2 4}$ & Met+Atorn=21 & p \\
\hline Age (year) & $46.8 \pm 7.4$ & $49.1 \pm 7.2$ & 0.32 \\
Male n, (\%) & $16(66.7)$ & $12(57.1)$ & 0.21 \\
Weight (kg) & $96.2 \pm 15.2$ & $89.6 \pm 12.0$ & 0.12 \\
BMI (kg/m²) & $33.6 \pm 6.2$ & $32.8 \pm 4.1$ & 0.62 \\
WC (cm) & $116.4 \pm 8.9$ & $114.1 \pm 8.12$ & 0.38 \\
VAI & $2.3 \pm 1.05$ & $2.51 \pm 1.0$ & 0.52 \\
Biochemical markers & & & \\
HbA1c (\%) & $8.1 \pm 1.7$ & $7.6 \pm 0.9$ & 0.14 \\
FSG (mmol/l) & $11.4 \pm 3.3$ & $11.2 \pm 2.1$ & 0.88 \\
TG (mmol/l) & $1.84 \pm 0.95$ & $1.9 \pm 0.7$ & 0.82 \\
TC (mmol/l) & $4.6 \pm 1.3$ & $4.7 \pm 1.2$ & 0.71 \\
HDL-C (mmol/l) & $1.3 \pm 0.4$ & $1.2 \pm 0.3$ & 0.9 \\
LDL-C (mmol/l) & $2.3 \pm 0.87$ & $2.2 \pm 0.9$ & 0.78 \\
\hline
\end{tabular}

Values are mean $\pm \mathrm{SD}, \mathrm{n}$ : The number of patients,

Met: Metformin, Ator: $10 \mathrm{mg}$ atorvastatin, BMI: Body mass

index, WC: Waist circumference, VAI: Visceral adiposity index,

HbA1c: Glycated hemoglobin, FSG: Fasting blood glucose, TG: Triglyceride,

TC: Total cholesterol, HDL-C: High-density lipoprotein cholesterol,

LDL-C: Low-density lipoprotein cholesterol, SD: Standard deviation

\section{Anthropometric measurements}

Body weight, wrist circumference, waist circumference (WC), and body mass index (BMI) were obtained for each participant at baseline and the end of the 90-day treatment.

\section{Measurement of the visceral adiposity index (VAI)}

The VAI was a gender-specific mathematical model based on simple anthropometric (BMI and WC) and functional parameters (triglyceride [TG] and high-density lipoprotein cholesterol [HDL-C]) and considered as an indicator of body fat distribution and function. It was calculated utilizing the formula of Amato et al. [16]. The distribution pattern of adipose tissue was corrected for TG and HDL-C levels to determine the VAI as follows:

Female VAI $=(\mathrm{WC} / 36.58+(1.89 \times \mathrm{BMI})) \times(\mathrm{TG} / 0.81) \times(1.52 / \mathrm{HDL}-\mathrm{c})$

Male VAI $=(\mathrm{WC} / 39.68+(1.88 \times \mathrm{BMI})) \times(\mathrm{TG} / 1.03) \times(1.31 / \mathrm{HDL}-\mathrm{c})$,

Where, WC is expressed in $\mathrm{cm}$, BMI in $\mathrm{kg} / \mathrm{m}^{2}$, TG in mmol/l, and HDL-c in $\mathrm{mmol} / \mathrm{l}$.

\section{Biochemical measurements}

Blood samples $(10 \mathrm{ml})$ were obtained by venipuncture from $12 \mathrm{~h}$ fasted patients at baseline (zero time) and after the 90-day treatment. Approximately $2.0 \mathrm{ml}$ of the blood was kept in EDTA containing tubes for the determination of glycated hemoglobin (HbA1c) by colorimetric methods (Roche-Cobas C 311; Roche Diagnostics GmbH, Mannheim, Germany). The remaining $8.0 \mathrm{ml}$ was drawn into plain tubes and allowed to clot before centrifuging at $\times 300 \mathrm{~g}$ for $20 \mathrm{~min}$ to obtain the serum. The serum was either analyzed immediately or stored at $-20^{\circ} \mathrm{C}$ for later use. The fasting serum glucose (FSG), TG, cholesterol, low-density lipoprotein cholesterol (LDL-C), HDL-C, creatinine levels, aspartate transaminase, and alanine aminotransferase activities were determined colorimetrically (Roche-Cobas C 311, Roche Diagnostics GmbH).

\section{Statistical analysis}

The data were statistically evaluated utilizing GraphPad Prism 5.1 software (GraphPad Software Inc., La Jolla, CA, USA). Continuous variables were presented as mean \pm standard deviation. Discrete variables were presented as numbers and frequencies. Discrete variables were analyzed using the Chi-square test, while paired t-test was used to evaluate the difference between baseline and day 90 values. Analysis of variance was used to determine the significant difference between means of independent samples, confirmed by Bonferroni's post hoc analysis. $\mathrm{p}<0.05$ was considered for statistical significance.

\section{RESULTS}

Table 1 shows no significant differences ( $p>0.05)$ between the baseline anthropometric characteristics and the biochemical markers of both treatment groups of the T2DM patients enrolled in the present study. In Table 2, treatment of the newly diagnosed T2DM patients with metformin alone produced significant reduction in the serum cholesterol level and the LDL/HDL ratio $(\mathrm{p}<0.05)$ compared with the corresponding baseline values, while the other components of lipid profile were not significantly changed ( $p>0.05)$. Moreover, the addition of $10 \mathrm{mg} /$ day of atorvastatin to the treatment protocol in the second group significantly improves all the lipid profile markers after 90 days compared with baseline values. However, analysis of data using ANOVA and post hoc test did not show significant differences between the two treatment groups after 90 days ( $p>0.05)$. (Fig. 1)

In the present study, Fig. 2 indicates that treatment with metformin alone significantly decreases all the adiposity markers (Fig. 3 a-d) compared with the baseline values $(p<0.05)$. Meanwhile, the combination of metformin with atorvastatin significantly improves these markers, except for the WC (Fig. 3a) compared with the baseline values. However, both treatment groups did not show significant differences between each other after 90 days of treatment (ANOVA, p>0.05). Regarding the impacts of adding atorvastatin to the metformin protocol, Fig. 3 indicates that in both groups of T2DM patients the glycemic control markers (FSG and HbA1c) were significantly decreased after 90 days of treatment $(\mathrm{p}<0.05)$ compared with their corresponding baseline values (Fig. 3a and b). However, both treatment approaches did not significantly differ from each other (ANOVA, $\mathrm{p}>0.05$ ) in this regard. In Table 3, the use of metformin alone did not negatively impact the markers of renal and hepatic functions after 90 days of treatment, compared with the baseline values; however, it significantly decreases serum glutamic-pyruvic transaminase (GPT) level compared with baseline. Meanwhile, the inclusion of atorvastatin produces a significant elevation of serum GPT and creatinine levels compared with baseline values $(\mathrm{p}<0.05)$; though the post-treatment values are still within the normal reference values.

\section{DISCUSSION}

In the present study, the use of metformin in newly diagnosed patients with T2DM improves body weight and glycemic control; however, the addition of low-dose atorvastatin to the treatment protocol did not improve these outcomes. Metformin, in a long-term study, reduces the risk of macrovascular disease after a follow-up period of 4.3 years [17], and this beneficial effect supports the clinical decision to continue metformin treatment in any patient with T2DM unless contraindicated. The result of the present study is consistent with that reported in an experimental animal model, which indicates that the combination of atorvastatin with metformin did not produce a better lipid-lowering effect than atorvastatin [18]. These clinical and experimental data are consistent with many previously published clinical and preclinical data $[17,19]$. In addition, Forouzandeh et al. reported that metformin markedly decreased atherosclerotic plaques without any effect on the cholesterol level in apoE-/- mice maintained on a high-fat diet [20]. These data may offer inconsistent idea regarding the addition of statins to metformin in newly diagnosed T2DM patients and may add more risks of adverse effects and cost burden.

The increased risk of impaired glucose metabolism due to the use of statins in T2DM patients was recognized in many reports; however, such therapeutic indication was not themselves major contributors to the increased risk of DM complications [10]. Moreover, the participants in such a type of studies who were prescribed statins already present with higher serum glucose levels at baseline, yet such observation cannot be used to explain the higher rates of DM among statin users [21]. In addition, our study indicated that $10 \mathrm{mg} /$ day did not increase the HbA1c and serum glucose levels, but there was no additional significant improvement in the studied markers when compared with the metformintreated group. However, Cheng et al. [22] reported a significant decrease 
in the clearance of serum glucose induced by treatment with atorvastatin at approximately $50 \mathrm{mg} / \mathrm{rabbit} /$ day for 16 weeks. Moreover, it was previously reported that statin-induced dysregulation of glycemic control is both dose- and time-dependent $[23,24]$.

When we analyzed the effect of metformin alone on the lipid profile,

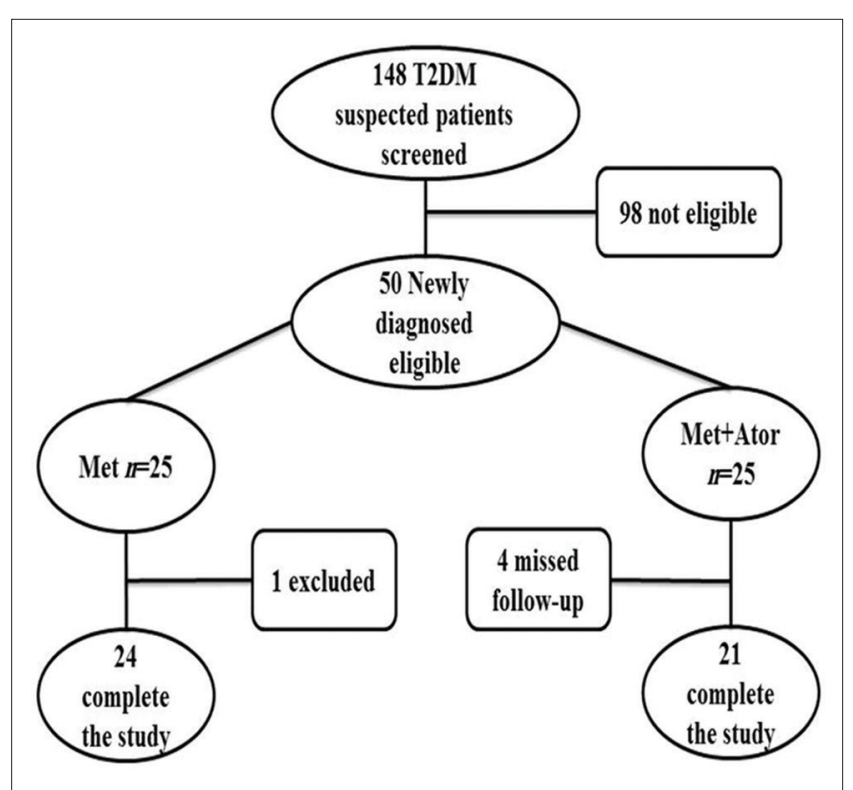

Fig. 1: Flowchart of the study; n: The number of patients; Met: Metformin; Ator: Atorvastatin our findings showed that only TG levels and LDL/HDL ratio were significantly improved; however, these effects were not significantly different compared with its combination with atorvastatin that improves all lipid profile components. These results indicated that the addition of atorvastatin with metformin did not influence the lipidlowering effects of monotherapy with metformin in newly diagnosed patients with T2DM. In previous studies, although metformin moderately improves the lipid profile, there were inconsistencies in its effects on the lipid parameters [7]. Moreover, many reports indicated that polymorphism in the OATP1B1 transporter gene may affect the area under the curve of oral antidiabetic agents [25]. Therefore, such uptake-transporter-mediated drug interactions may be involved in the differential effects of atorvastatin that may influence metformin response, which is not clearly revealed due to the short duration of the study. Importantly, other reports have shown that the atorvastatin-induced impairment of glycemic control was completely reversed by metformin [26]; this may explain the non-significant differences reported in the present study between the two groups of T2DM patients. Accordingly, the addition of atorvastatin to metformin therapy in newly diagnosed T2DM patients with a relatively normal lipid profile may be irrational and cost ineffective and the emergence of adverse effects may be highly expected with long-term use. Moreover, our results of the safety profile for metformin/atorvastatin combination treatment indicate that the addition of atorvastatin may negatively impact the function of the liver and/or kidneys, which is consistent with previous reports $[27,28]$. It has been reported that atorvastatin is more effective in reducing the atherogenic index in responders of metformin than in non-responders, which may indicate higher atherogenic characteristics in the non-responders. Accordingly, newly diagnosed patients with T2DM who show inadequate response to metformin may need better treatment approaches to lower atherogenic lipids. According to a program
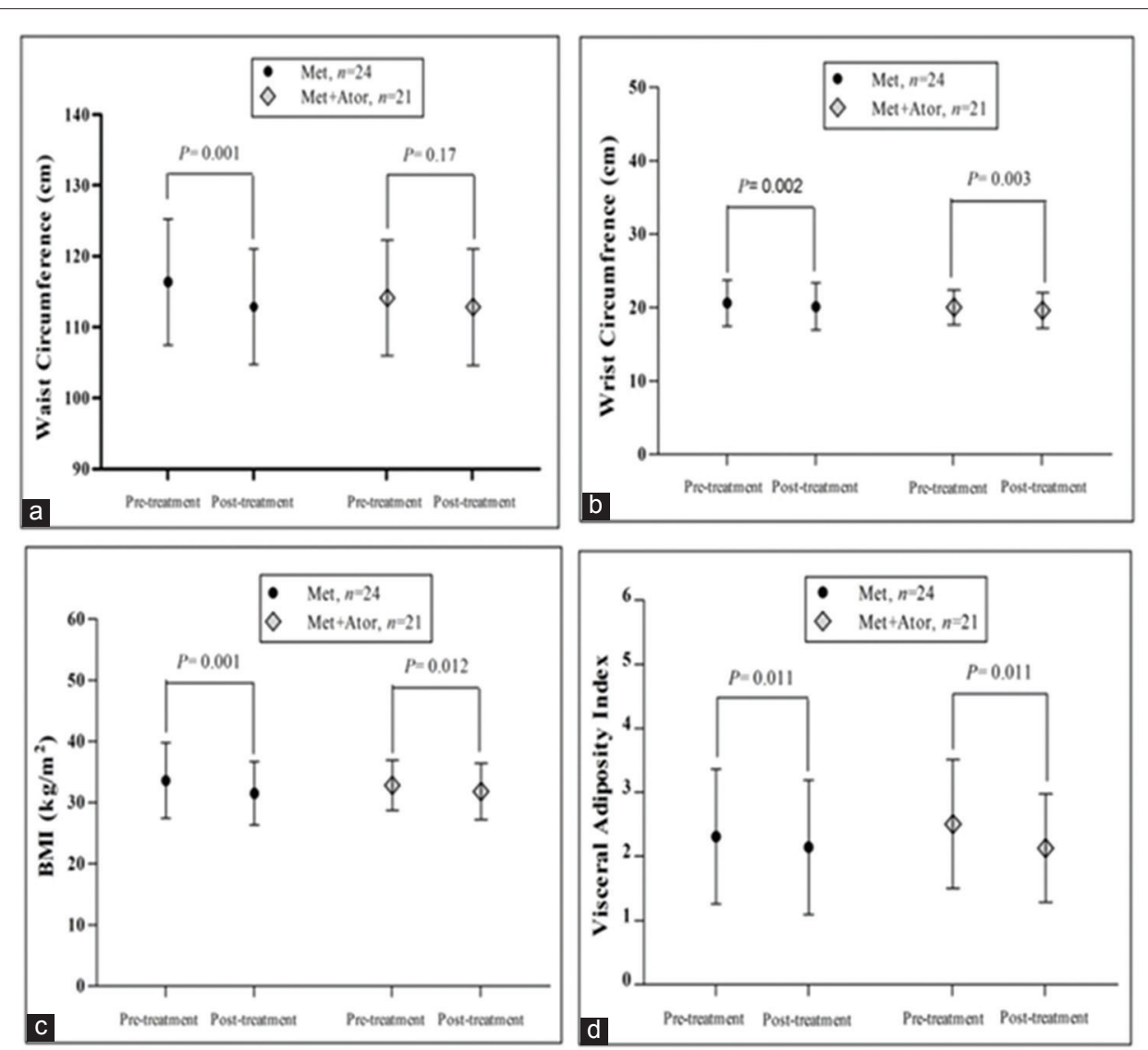

Fig. 2: Effects of metformin alone or its combination with atorvastatin on the markers of adiposity: (a) Waist circumference; (b) wrist circumferences; (c) body mass index; and (d) visceral adiposity index 


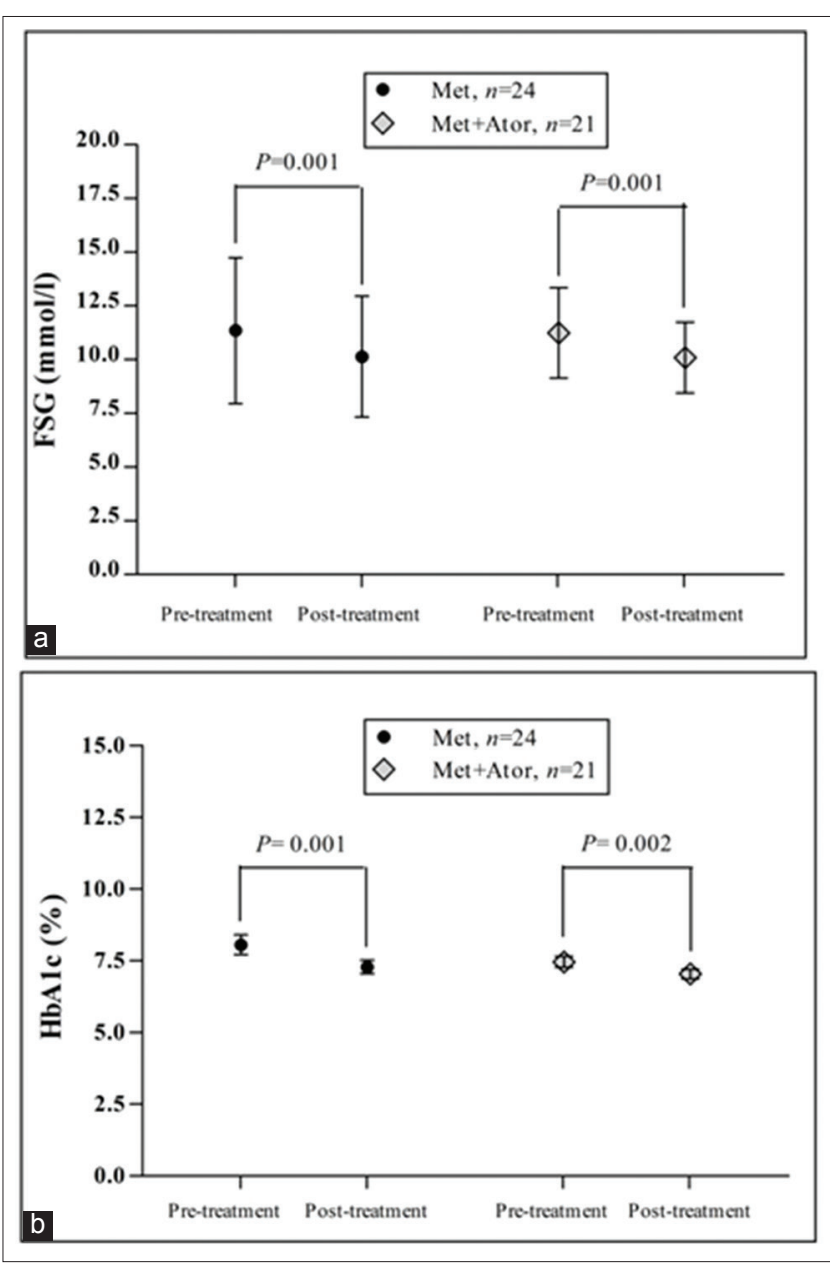

Fig. 3: Effects of metformin alone or its combination with atorvastatin on the glycemic control markers: (a) Fasting serum glucose and (b) glycated hemoglobin

Table 2: Effects of metformin alone or its combination with atorvastatin on the lipid profile markers

\begin{tabular}{|c|c|c|c|c|}
\hline \multirow[t]{2}{*}{ Parameters } & \multicolumn{2}{|c|}{ Met $(n=24)$} & \multicolumn{2}{|c|}{ Met+Ator $(n=21)$} \\
\hline & Baseline & 90 days & Baseline & 90 days \\
\hline TG (mmol/l) & $1.84 \pm 0.95$ & $1.8 \pm 0.9^{*}$ & $1.9 \pm 0.7$ & $1.7 \pm 0.5^{*}$ \\
\hline $\mathrm{TC}(\mathrm{mmol} / \mathrm{l})$ & $4.6 \pm 1.3$ & $4.4 \pm 1.2$ & $4.7 \pm 1.2$ & $4.2 \pm 1.1^{*}$ \\
\hline LDL-C (mmol/l) & $2.3 \pm 0.87$ & $2.2 \pm 1.0$ & $2.2 \pm 0.9$ & $1.9 \pm 0.8^{*}$ \\
\hline HDL-C (mmol/l) & $1.3 \pm 0.4$ & $1.4 \pm 0.35$ & $1.2 \pm 0.3$ & $1.43 \pm 0.3^{*}$ \\
\hline LDL-C/HDL-C & $1.77 \pm 0.9$ & $1.6 \pm 0.7^{*}$ & $1.8 \pm 0.7$ & $1.3 \pm 0.6^{*}$ \\
\hline
\end{tabular}

Values are mean \pm SD; $\mathrm{n}$ : The number of patients. ${ }^{*}$ Significantly different from baseline in each group (paired t-test, $\mathrm{P}<0.05$ ), Met: Metformin, Ator: $10 \mathrm{mg}$ atorvastatin, TG: Triglyceride, TC: Total cholesterol, LDL-C: Low-density lipoprotein cholesterol, HDL-C: High-density lipoprotein cholesterol, SD: Standard deviation

suggested in a review article [29], niacin or high-dose omega-3 fatty acid supplementation could be used in newly diagnosed T2DM patients with borderline values of lipid profile, secondary to lifestyle modifications before using a potent statin such as atorvastatin as the first treatment priority.

\section{CONCLUSION}

According to our findings, the addition of $10 \mathrm{mg} /$ day atorvastatin with metformin in the treatment of newly diagnosed T2DM patients did not produce significant improvement in glycemic control, adiposity index, and lipid profile compared with the use of metformin alone. The impact
Table 3: Effects of metformin alone or its combination with atorvastatin on the liver and kidney function markers

\begin{tabular}{|c|c|c|}
\hline \multirow[t]{2}{*}{ Parameters } & Met $(n=24)$ & Met+Ator $(n=21)$ \\
\hline & Baseline 90 days & Baseline 90 days \\
\hline GOT (U/L) & $19.3 \pm 7.1 \quad 18.7 \pm 6.8$ & $19.1 \pm 7.1 \quad 18.5 \pm 4.1$ \\
\hline GPT (U/L) & $15.9 \pm 4.9 \quad 12.8 \pm 5.9 *$ & $15.7 \pm 5.317 .6 \pm 6.5^{*}$ \\
\hline Serum creatinine $(\mathrm{mmol} / \mathrm{l})$ & $60.1 \pm 2.6 \quad 62.7 \pm 4.8$ & $63.6 \pm 5.0 \quad 69.8 \pm 4.7^{*}$ \\
\hline
\end{tabular}

Values are mean \pm SD; n: The number of patients, *significantly different from baseline in each group (paired t-test, $\mathrm{P}<0.05$ ), Met: Metformin, Ator: $10 \mathrm{mg}$ atorvastatin, GOT: Glutamic-oxaloacetic transaminase, GPT: Glutamic-pyruvic transaminase, SD: Standard deviation

of the combination on the liver and kidney functions should be strongly considered.

\section{ACKNOWLEDGMENT}

The authors thank Al-Rafidain University College for supporting the project.

\section{AUTHORS' CONTRIBUTION}

Dr. Hussain SA - contributed to the selection of topic, research concept development, and guided the research process and manuscript preparation. Dr. Mohammed SS - conducted the study and participated in data processing. Dr. Mustafa WW - conducted the study and participated in data collection and manuscript writing.

\section{CONFLICTS OF INTEREST STATEMENT}

The authors declare that they have no conflicts of interest.

\section{REFERENCES}

1. Moradi M, Mousavi S. Drug use evaluation of diabetes mellitus in nonhospitalized patients. Int J Pharm Pharm Sci 2016;8:337-41.

2. Ramanna MK, Ruckmani A, Janti SS, Eerike M, Prabu RL. Burden of therapy in patients suffering from diabetes mellitus and hypertension. Int J Pharm Pharm Sci 2017;9:210-5.

3. Athyros VG, Kakafika AI, Tziomalos K, Karagiannis A, Mikhailidis DP. Pleiotropic effects of statins: Clinical evidence. Curr Pharm Des 2009; 15:479-89.

4. Okopien B, Krysiak R, Kowalski J, Madej A, Belowski D, Zielinski M, et al. Monocyte release of tumor necrosis factor-alpha and interleukin1beta in primary Type IIa and IIb dyslipidemic patients treated with statins or fibrates. J Cardiovasc Pharmacol 2005;46:377-86.

5. Pruski M, Krysiak R, Okopien B. Pleiotropic action of short-term metformin and fenofibrate treatment, combined with lifestyle intervention, in Type 2 diabetic patients with mixed dyslipidemia. Diabetes Care 2009;32:1421-4.

6. Bulcao C, Ribeiro-Filho FF, Sanudo A, Ferreira SG. Effects of simvastatin and metformin on inflammation and insulin resistance in individuals with a mild metabolic syndrome. Am J Cardiovasc Drugs 2007; 7:219-4.

7. Buse JB, Tan MH, Prince MJ, Erickson PP. The effects of oral antihyperglycemic medications on serum lipid profiles in patients with Type 2 diabetes. Diabetes Obes Metab 2004;6:133-56.

8. Costa J, Borges M, David C, Carneiro AV. Efficacy of lipid lowering drug treatment for diabetic and nondiabetic patients: A meta-analysis of randomized controlled trials. BMJ 2006;332:1115-24.

9. Owusu ES, Samanta M, Shaw JE, Majeed A, Khunti K, Paul SK. Weight loss and mortality risk in patients with different adiposity at diagnosis of Type 2 diabetes: A longitudinal cohort study. Nutr Diabetes 2018;8:37.

10. Crandall JP, Mather K, Rajpathak SN, Goldberg RB, Watson K, Foo S, et al. Statin use and risk of developing diabetes: Results from the diabetes prevention program. BMJ Open Diabetes Res Care 2017;5:e000438.

11. Macedo AF, Douglas I, Smeeth L, Forbes H, Ebrahim S. Statins and the risk of Type 2 diabetes mellitus: Cohort study using the UK clinical practice search datalink. BMC Cardiovasc Disord 2014;14:85.

12. Bosi E. Metformin-the gold standard in Type 2 diabetes: What does the evidence tell us? Diabetes Obes Metab 2009;11:3-8 
13. Mahrooz A, Parsanasab H, Hashemi-Soteh MB, Kashi Z, Bahar A, Alizadeh A, et al. The role of clinical response to metformin in patients newly diagnosed with Type 2 diabetes: A monotherapy study. Clin Exp Med 2015;15:159-65.

14. Ridker PM, Danielson E, Fonseca FA, Genest J, Gotto AM, Kastelein JJ, et al. Rosuvastatin to prevent vascular events in men and women with elevated C-reactive protein. N Engl J Med 2008;359:2195-207.

15. Bennet PH. Impact of the new WHO classification and diagnostic criteria. Diabetes Obes Metab 1999;1 Suppl 2:S1-6.

16. Amato MC, Giordano C, Galia M, Criscimanna A, Vitabile S, Midiri M, et al. Visceral adiposity index: A reliable indicator of visceral fat function associated with cardiometabolic risk. Diabetes Care 2010;33:920-2.

17. Kooy A, de Jager J, Lehert P, Bets D, Wulffelé MG, Donker AJ, et al. Long-term effects of metformin on metabolism and microvascular and macrovascular disease in patients with Type 2 diabetes mellitus. Arch Intern Med 2009;169:616-25.

18. Luo F, Guo Y, Ruan GY, Long JK, Zheng XL, Xia Q, et al. Combined use of metformin and atorvastatin attenuates atherosclerosis in rabbits fed a high-cholesterol diet. Sci Rep 2017;7:2169.

19. Preiss D, Lloyd SM, Ford I, McMurray JJ, Holman RR, Welsh P, et al. Metformin for non-diabetic patients with coronary heart disease (the CAMERA study): A randomized controlled trial. Lancet Diabetes Endocrinol 2014;2:116-24.

20. Forouzandeh F, Salazar G, Patrushev N, Xiong S, Hilenski L, Fei B, et al. Metformin beyond diabetes: Pleiotropic benefits of metformin in attenuation of atherosclerosis. J Am Heart Assoc 2014;3:e01202.
21. Knowler WC, Barrett-Connor E, Fowler SE, Hamman RF, Lachin JM, Walker EA, et al. Reduction in the incidence of type 2 diabetes with lifestyle intervention or metformin. N Engl J Med 2002;346:393-403.

22. Cheng D, Wang Y, Gao S, Wang X, Sun W, Bai L, et al. Atorvastatin delays the glucose clearance rate in hypercholesterolemic rabbits. Biomed Pharmacother 2015;72:24-9.

23. Cederberg H, Stančáková A, Yaluri N, Modi S, Kuusisto J, Laakso M. Increased risk of diabetes with statin treatment is associated with impaired insulin sensitivity and insulin secretion: A 6-year follow-up study of the METSIM cohort. Diabetologia 2015;58:1109-17.

24. Wang KL, Liu CJ, Chao TF, Chen SJ, Wu CH, Huang CM, et al. Risk of new-onset diabetes mellitus versus reduction in cardiovascular events with statin therapy. Am J Cardiol 2014;113:631-6.

25. König J, Seithel A, Gradhand U, Fromm MF. Pharmacogenomics of human OATP transporters. Naunyn Schmiedebergs Arch Pharmacol 2006;372:432-43.

26. Krysiak R, Okopien B. The effect of metformin on monocyte secretory function in simvastatin-treated patients with impaired fasting glucose. Metabolism 2013;62:39-43.

27. Björnsson ES. Hepatotoxicity of statins and other lipid-lowering agents. Liver Int 2017;37:173-8

28. Nasri H, Hasanpour Z, Nematbakhsh M, Ahmadi A, Rafieian-Kopaei M. The effect of the various doses of atorvastatin on renal tubular cells; an experimental study. J Nephropathol 2016;5:111-5.

29. Rubenfire M, Brook RD, Rosenson RS. Treating mixed hyperlipidemia and the atherogenic lipid phenotype for prevention of cardiovascular events. Am J Med 2010;123:892-8. 